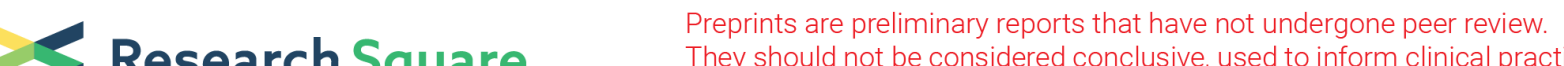 $\begin{array}{ll}\text { Research Square } & \text { They should not be considered conclusive, used to inform clinical practice, } \\ \text { or referenced by the media as validated information. }\end{array}$
}

\section{Comparison of extended reality and conventional methods of basic life support training: protocol for a multinational pragmatic clinical trial (XR BLS trial)}

Chang Woo Im

Seoul National University Bundang Hospital https://orcid.org/0000-0002-9469-8977

Dong Keon Lee

Seoul National University Bundang Hospital

You Hwan Jo ( $\sim$ emdrjyh@gmail.com )

Seoul National University Bundang Hospital

\section{Todd Chang}

Children's Hospital Los Angeles Department of Pediatrics

Joo Lee Song

Children's Hospital Los Angeles Department of Pediatrics

Cindy Luu

Children's Hospital Los Angeles Department of Pediatrics

\section{Ralph Mackinnon}

Royal Manchester Children's Hospital

Kuyt Katherine

Royal Manchester Children's Hospital

\section{Suresh Pillai}

National University of Singapore

\section{Chuen Neng Lee}

National University of Singapore

\section{Sanghoon Jheon}

Seoul National University Bundang Hospital

\section{Soyeon Ahn}

Seoul National University Bundang Hospital

\section{Seung Hyun Won}

Seoul National University Bundang Hospital

\section{Study protocol}

Keywords: Cardiopulmonary Resuscitation, Basic Life Support, Virtual Reality, Extended Reality, Education 
Posted Date: April 27th, 2021

DOl: https://doi.org/10.21203/rs.3.rs-462904/v1

License: (c) (i) This work is licensed under a Creative Commons Attribution 4.0 International License. Read Full License 


\section{Abstract}

\section{Background}

Conventional cardiopulmonary resuscitation (CPR) training for the general public involves the use of a manikin and a training video, which has limitations of lack of realism and immersion. To overcome these limitations, virtual reality and extended reality technologies are being used in the field of medical education. The aim of this study is to explore the efficacy and safety of the extended reality (XR)-based basic life support (BLS) training.

\section{Methods}

This study is a prospective, multinational, multicentre, randomized controlled study. Four institutions in 4 countries will participate in the study. A total of 154 participants will be randomly assigned to either the $\mathrm{XR}$ group or the conventional group stratified by institution and sex (1:1 ratio). Each participant who is allocated to either group will be sent to a separate room to receive training with an XR BLS module or conventional CPR training video. All participants will perform a test on a CPR manikin after the training.

The primary outcome will be mean compression depth. The secondary outcome will be overall BLS performances, including compression rate, correct hand position, compression and full release and hands-off time.

\section{Discussion}

Using VR to establish a virtual educational environment can give trainees a sense of realism. In the XR environment, which combines the virtual world with the real world, trainees can more effectively learn various skills. This trial will provide evidence of the usefulness of XR in CPR education.

Trial registration

ClinicalTrials.gov NCT04736888. Registered on 29 January 2021

\section{Introduction}

\section{Background and rationale}

In Europe and the United States, more than 300,000 cases of out-of-hospital cardiac arrest (OHCA) occur annually, and OHCA is a significant global health problem [4, 5]. To improve the outcomes of patients with OHCA, the early recognition of cardiac arrest and performance of cardiopulmonary resuscitation (CPR) by bystanders are the most essential steps. However, bystander CPR is provided to patients with OHCA in less than $50 \%$ of cases [6, 7]. Therefore, it is very important to provide CPR training to the general public. 
Conventional CPR training is based on the use of a manikin and a training video. Though several feedback devices have been developed to improve the effectiveness of the training, they were neither realistic nor immersive [8]. In addition, in conventional training programs, trainees are constrained in terms of time and location, as they are usually kept to a schedule.

Virtual reality (VR) technology, which was designed to maximize immersion, could be used to overcome those limitations, which in turn may improve the effectiveness of CPR training. However, even with VR technology, procedures such as chest compressions, ventilation, and defibrillation cannot be implemented as in the real world [9]. Extended reality (XR), which combines the virtual and real worlds, could overcome these limitations by facilitating the use of real-world manikins in the virtual environment.

Objectives

The aim of this study is to compare the efficacy of the XR-based basic life support (BLS) training method, which combines VR and the use of manikins, with that of the conventional training method. The hypothesis of this study is that the BLS skills gained by the XR method are not inferior to those gained by the conventional method.

Trial design

This study is a prospective, multinational, multicentre, randomized controlled study. Four institutions in 4 countries will participate in the study. The trial protocol will be approved by the Institutional Review Boards of the individual participating institutions (Fig. 1). This study was registered at ClinicalTrials.gov (NCT number: NCT04736888), and the protocol has been designed following the guidelines of the Consolidated Standards of Reporting Trials and Standard Protocol Items: Recommendations for Interventional Trials (SPIRIT) (see Supplementary file 1 SPIRIT 2013 checklist).

\section{Methods: Participants, Interventions, And Outcomes Study setting}

We designed a multinational, multicentre, randomized clinical trial that will include 154 participants from Seoul National University Bundang Hospital (South Korea), Children's Hospital Los Angeles (USA), Royal Manchester Children's Hospital (UK), and National University of Singapore (Singapore).

CBS 2.0 TM (Tetra Signum, Inc., Seoul, South Korea), a self-learning type of XR solution, will be used for the XR-based BLS training. A total of 154 participants will be enrolled after recruitment is announced, and a stratified permuted block will be used for randomization. We plan to begin trial recruitment in March 2021 , and the planned study period will be six months.

\section{Eligibility criteria}


Individuals who are not healthcare providers and are 18 years old or older will be eligible for inclusion. Participants who are not capable of performing either the training or the CPR test due to physical or cognitive limitations will be excluded [10-13]. Those who have upper extremity injuries or are pregnant will also be excluded [14]. If participants experience dizziness, headache, or motion sickness during the 2minute XR device adaptation period that prevents them from participating in the simulation study, they will also be excluded [15].

\section{Ethics approval}

The investigators of each institution will explain to the participants the purpose of the study, the risks and benefits of the research, and how to contact the study investigators (or the Seoul National University Bundang Hospital ethics review board) regarding problems that occurred in this study prior to obtaining their informed consent.

\section{Interventions}

\section{Explanation of the choice of comparators}

Participants will be randomly assigned to (1) the XR group or (2) the conventional group stratified by institution and sex. Because watching videos and following their instructions is the current standard CPR training method, the conventional group has been chosen as the control group.

\section{Intervention description}

Participants will be enrolled competitively, but a minimum of 32 participants will be enrolled from each participating institution to minimize racial and physical differences among the participants in each country. Participants will be randomly assigned to either the XR group or the conventional group (1:1 ratio), with randomization stratified by institution and sex. The randomization sequence will be generated in SAS software (version 9.4; SAS Institute Inc., Cary, North Carolina, USA) by an independent statistician and the randomization procedure will be implemented in REDCap software (version 9.5.2, Vanderbilt University, Nashville, TN, USA). Each participant who is allocated to either group will be sent to a separate room (room A for the XR group and room B for the conventional group) and trained with an XR BLS module or conventional CPR training video.

Conventional CPR training consists of a BLS video and a manikin equipped with a feedback device. The XR group participants, on the other hand, will be provided training via the XR BLS module and are allotted an additional 2 minutes that is needed to adapt to the XR equipment. In both groups, BLS training will take approximately 60 minutes and is composed of checking responsiveness, calling for help, checking breathing, performing chest compressions, performing rescue breathing, and using a defibrillator. 
Each group will be trained to perform both standard 30:2 CPR and hands-only CPR. Participants will be taught to use a face mask or cloth to cover the patient's face and to perform hands-only CPR to reduce the risk of disease transmission when the patient is a suspected or confirmed or unknown case of COVID$19[16,17]$. In addition, participants will be taught to perform standard 30:2 CPR, too. The XR group will follow the instructions of the XR content, while the conventional group will watch the BLS video and follow its instructions [18].

All participants and BLS instructors will wear a mask and follow social distancing guidelines to prevent the transmission of respiratory diseases during the study. Both groups will have a 5-minute Q\&A (question and answer) session after training (Fig 1).

\section{Test procedure}

All participants will take a test at least 30 minutes after completing the training $[11,12]$. The test will be conducted by a BLS instructor, who has not been involved in BLS training. Laerdal Resusci Anne ${ }^{\text {TM }}$ manikin (Laerdal Medical Corporation, Stavanger, Norway) will be used. Additionally, depending on the policies of each participating institution, the test will be recorded on a smartphone, video camera, or other recording devices after obtaining consent from the participant.

The test will consist of two parts. Each part will assess the CPR steps of 'checking for a response', 'calling for help', 'checking for breathing', 'performing chest compressions', and 'using an automated electrical defibrillator (AED)'. This study's main test, Test \#1, will use hands-only CPR as the primary assessment criteria and will be conducted in line with the American Heart Association (AHA) and European Resuscitation Council (ERC)'s 'Basic life support guidelines for adults with suspected or confirmed COVID$19^{\prime}$.

Test \#2 will assume a scenario in which the patient is not suspected of having COVID-19 and will assess BLS skills with the $30: 2$ of chest compression to rescue breath ratio. However, as face shields and pocket masks do not guarantee protection from the transmission of COVID-19 between the participants via a manikin, the rescue breaths will be substituted by a 10 -second rest. To reduce interference between the two tests, after the continuous chest compression assessment (Test \#1), the assessment of 5 cycles of 30 chest compressions with a 10-second rest between each cycle as a substitute for rescue breaths (Test \#2) will be conducted after a 10-minute break. To evaluate the effectiveness of BLS training, a Laerdal Resusci Anne ${ }^{\text {TM }}$ manikin connected to the Laerdal SimPad SkillReporter ${ }^{T M}$ will be used. All records stored on the Skillreporter ${ }^{\mathrm{TM}}$ will be analysed on a computer equipped with the Laerdal PC Skill Reporting System (Laerdal Medical Corporation, Stavanger, Norway). After the test, all participants will be asked to fill out a questionnaire.

\section{Criteria for discontinuing or modifying the allocated interventions}


During the study, those who experience dizziness, headache, motion sickness, or physical injuries that prevent their participation in this study will be excluded [15]. Participants may choose to discontinue their participation at any time for any reason.

\section{Provisions for post-trial care}

This study investigates the efficacy and safety of the XR-based BLS training method. The probability of side effects during CPR training is very low. If any side effects occur during BLS training, appropriate treatment will be provided by each institution. In the case of serious side effects related to this study, the research director will follow each institution's IRB protocol for reporting and subsequent measures required.

\section{Outcomes}

\section{Primary outcomes}

The primary outcome will be the mean compression depth $(\mathrm{mm})$ over 2 minutes, which will be measured in Test \#1, during which continuous chest compression will be delivered.

\section{Secondary outcomes}

The secondary outcomes will be as follows: the total number of chest compressions (n), the mean chest compression depth of each 30-second epoch, correct hand position (\%), adequate compression depth (\%), compression and full release (\%), mean compression rate (number per minute), adequate compression rate (\%), adequate compression depth and rate (\%), and hands-off time (sec).

In addition, the successful implementation of the steps involving checking for a response, calling for help and checking for breathing will be compared. Other test results include the time interval from arrival on the scene (patient contact in the simulation) to the first chest compression (sec), the total number of chest compressions with full release, correct hand position (total number of times) and correct AED use. For institutions where the policies allow recordings, the time interval from arrival on the scene (patient contact in the simulation) to the first chest compression (sec), time from powering on the AED to defibrillation (sec) and the time from checking for a response to defibrillation (sec) will be assessed by reviewing the recordings. Regarding the questionnaire (supplementary file \#2,3), a 5-point Likert scale will be used to assess the effectiveness of training, fatigue level, and realism ( 1 = strongly disagree, $5=$ strongly agree) [1-3]. The demographics of participants, such as age, sex, weight, height and status of previous CPR training, will also be investigated. 


\section{Participant timeline}

The participant timelines are presented in Fig. 2.

\section{Sample size}

This is a non-inferiority clinical trial that compares the effectiveness of XR BLS training to that of conventional BLS training, which is based on a video. To calculate the sample size needed to assess the primary outcome of the mean number of chest compressions, which is determined in Test \#1, we defined a non-inferiority margin on the basis of previous studies $[10,12,13,19,20]$. Since conventional training increased chest compression depth by $5 \mathrm{~mm}$ and a decrease in depth by $5 \mathrm{~mm}$ was reported to be associated with reduced survival after cardiac arrest $[19,20]$, the non-inferiority margin was defined as -5 $\mathrm{mm}$. With an expected standard deviation of $10 \mathrm{~mm}[12,13,19]$, a one-sided alpha of $5 \%$, and a power of $90 \%$, each group needed 69 participants. After adjusting for a 10\% drop-out rate, 77 participants are needed in each group.

\section{Recruitment}

Volunteers will be recruited through announcements published on the official website of each institution as well as via direct contact or sending emails. Participants will be enrolled competitively, but a minimum of 32 participants will be enrolled from each participating institution. Recruitment will be completed once the required number of volunteers has been obtained. There will be no official paid incentives to participate in this study, but small gifts may be provided depending on the institutional conditions.

\section{Assignment of interventions: allocation}

\section{Sequence generation}

Random numbers will be generated with the stratified randomized permuted block design generated by SAS software and the randomization procedure will be implemented in REDCap software. Institution and sex will be applied as the stratification factors, and REDCap will randomly allocate participants to the XR or conventional group.

\section{Concealment mechanism}

REDCap will use its algorithm for randomization, which is unknown to the investigators. 


\section{Implementation}

Not applicable

\section{Data collection and management}

\section{Data management}

The answers to the survey questions will be entered directly into REDCap or provided on paper and then entered into REDCap. REDCap is the software toolset and workflow methodology for electronic data collection and management. The person in charge at each institution will be able to access only their institution's data. If the values that are entered are duplicated or out of range, members of the data monitoring committee will confirm them (Table 1). REDCap servers are managed by Seoul National University Bundang Hospital. All web-based information transmission will be encrypted. All protocols, consent forms, and data will be stored in the REDCap system. Data monitoring will be performed by an independent data coordinating centre. Two independent statisticians who will not participate in the actual simulation study will be able to access the trial dataset. The collected data will be stored for up to 6 months after the end of the study by the independent data coordinating centre. When it is necessary to store data for a longer period, the data could be sent to the research administration team to be stored for up to 3 years.

\section{Confidentiality}

No personally identifiable information will be stored. Other data will be discarded after this study.

\section{Statistical methods}

\section{Statistical analysis of the primary and secondary outcomes}

Data will be analysed using SPSS 25.0 (IBM SPSS, Inc., Chicago, IL, USA) and Stata 10.0 (StataCorp, College Station, Texas, USA). The primary outcome will be assessed for non-inferiority using a 1-sided $95 \%$ confidence interval of the mean compression depth between the XR and conventional group. If the lower limit of the confidence interval is above the pre-specified non-inferiority limit -5 , then the noninferiority of XR to conventional will be established. Continuous variables will be presented as the means \pm standard deviations (SDs) or medians with interquartile ranges (IQRs) after the normality of their distributions have been assessed with the Shapiro-Wilk test. The chi-square test or Fisher's exact test will be used for comparisons of nominal variables, while the independent t-test and the Mann-Whitney U test will be used to compare continuous variables. 


\section{Interim analyses}

Not applicable

\section{Methods for additional analyses}

Not applicable

\section{Oversight and monitoring}

\section{Composition of the coordinating centre and trial steering committee}

A team at Seoul National University Bundang Hospital and peer reviewers from Children's Hospital Los Angeles, Royal Manchester Children's Hospital and National University of Singapore will constantly monitor the study datasets and implementation and provide recommendations for the discontinuation of any or all of the study or the modification of any aspect of the protocol.

Their roles include the preparation of the protocol and any revisions, the application to obtain approval from the Research Ethics Committee, and the publication of study reports.

\section{Composition of the data monitoring committee, its role and reporting structure}

The data monitoring committee will consist of one investigator in charge at each institution, including one statistician at Seoul National University Bundang Hospital, who will not be involved in the actual simulation study. If the entered values are duplicated or are out of range, the data monitoring committee will check and correct the values.

\section{Adverse event reporting and harms}

Any adverse events occurring in the study will be reported to the Institutional Review Board. If there is an adverse event that prevents a participant from completing the study, the participant will be withdrawn, and the investigators will provide all necessary support and treatment.

\section{Plans for auditing trial conduct and audit frequency}


Because this study is intended to be completed within 6 months, we do not have a plan for auditing the conduct of the trial.

\section{Plans for communicating important protocol amendments to relevant parties}

If there are important protocol modifications, all investigators will be notified immediately via e-mail.

\section{Dissemination plans}

We will disseminate our findings through journal publications and conference presentations. If participants want to receive a report of the study results, we will send it via email after the study is complete.

\section{Discussion}

In industrialized countries, OHCA is one of the most common causes of death; every year, 1 in 1000 people die from OHCA [17]. Despite many attempts to improve the survival rate, it is still between $6.7 \%$ and $8.4 \%$ [18].

According to the International Liaison Committee on Resuscitation (ILCOR), bystander CPR is associated with an improved survival rate and neurological recovery [19-21]. Previous research found that bystander CPR was more likely to be performed when laypersons had participated in CPR training [24]. Hence, increased layperson CPR training is related to an increase in the survival rate after $\mathrm{OHCA}$, including better neurological outcomes [22,23].

Conventional CPR training consists of checking for a response, calling for help, checking for breathing, performing chest compressions, performing rescue breathing, and using an AED. In conventional CPR training, the trainees watch the video and follow the instructions on the manikins [25], and BLS instructors guide trainees throughout the training process. Although this training method helped increase the bystander CPR rate, it has limitations due to its lack of realism and immersion. Moreover, the emergence of COVID-19 has necessitated training that is not performed face-to-face.

VR is one of the solutions that could overcome these limitations. It has been used in CPR training to maximize realism and immersion, which could improve the effectiveness of training. Nas et al.[2] reported that VR training resulted in a similar chest compression rate to conventional training. However, VR also has critical limitations. Procedures such as chest compressions, ventilations, and defibrillations cannot be implemented as in real environments in VR settings[1]. Although Nas et al.[2] used a pillow to practice chest compression in VR, it was soft enough to be compressed more easily than Manikin, which might result in a more shallow compression depth in the VR training group compared to the conventional group. 
Recently, extended reality (XR), which combines the virtual and real worlds, was introduced. It could overcome the limitations of VR by embedding a real-world manikin into virtual environments, enabling trainees to actually practice chest compressions, rescue breaths, and defibrillation with the manikins.

The aim of this study was to compare the validity and efficacy of the XR-based BLS training method to those of the conventional training method. In the future, there will be major changes in the education field because of advanced technologies. Especially after the COVID-19 pandemic, the demand for remote education will increase, and CPR will be included in this trend. This study will be meaningful as a guide for future CPR training methods.

\section{Trial status}

The recruitment of participants for this study will start in June 2021.

Recruitment is expected to be completed in September 2021.

Current protocol version and date: V1.0, 11th April 2021.

\section{Abbreviations}

VR

Virtual Reality

$\mathrm{XR}$

Extended Reality

CPR

Cardiopulmonary Resuscitation

BLS

Basic Life Support

REDCap

Research Electronic Data Capture

RCT

Randomized Clinical Trial

\section{Declarations}

\section{Authors' contributions}

Dong-Hyun Jang: Investigation, Formal analysis, Writing - Original Draft

Dong Keon Lee: Conceptualization, Methodology, Writing - Review \& Editing

Jonghwan Shin: Project administration, Data Curation 
Gil Joon Suh: Supervision

Woon Yong Kwon: Project administration

You Hwan Jo: Project administration, Validation

Seung Min Park: Data Curation

Huijai Lee: Validation

Yoon Sun Jung: Data Curation

\section{Acknowledgements}

Thank you to the participants who will participate in the study. Thanks to Tetra Signum for providing the equipment free of charge.

\section{Funding}

This work is partly supported by a National Research Foundation of Korea (NRF) grant funded by the Korean government (MSIT) (No. NRF-2019R1F1A1059719) and by the Seoul National University Bundang Hospital Research Fund (No. 14-2019-021).

\section{Availability of data and material}

After the completion of the study, the results will be disseminated through conferences and scientific publications in journals related to emergency medicine, resuscitation medicine, and medical education.

\section{Ethics approval and consent to participate}

Human Research Ethics Committee approval was obtained from the Seoul National University Bundang Hospital ethics review board on 24 December 2020. Number: B-2012-657-301. Written informed consent will be obtained from all trainees prior to their participation.

\section{Consent for publication}

Not applicable.

\section{Competing interests}


You Hwan Jo and Sanghoon Jheon hold unlisted shares of Tetra Signum Inc. They will not get access to the study data, and will not participate in data analysis.

\section{References}

1. Semeraro F, Frisoli A, Loconsole C, Bannò F, Tammaro G, Imbriaco G, et al. Motion detection technology as a tool for cardiopulmonary resuscitation (CPR) quality training: a randomised crossover mannequin pilot study. Resuscitation. 2013;84:501-7.

2. Semeraro F, Taggi F, Tammaro G, Imbriaco G, Marchetti L, Cerchiari EL. iCPR: a new application of high-quality cardiopulmonary resuscitation training. Resuscitation. 2011;82:436-41.

3. Semeraro F, Frisoli A, Bergamasco M, Cerchiari EL. Virtual reality enhanced mannequin (VREM) that is well received by resuscitation experts. Resuscitation. 2009;80:489-92.

4. Iwashyna TJ. Survivorship Will Be the Defining Challenge of Critical Care in the 21 st Century [Internet]. Annals of Internal Medicine. 2010. p. 204. Available from: http://dx.doi.org/10.7326/00034819-153-3-201008030-00013

5. Gräsner J-T, Lefering R, Koster RW, Masterson S, Böttiger BW, Herlitz J, et al. EuReCa ONE-27 Nations, ONE Europe, ONE Registry: A prospective one month analysis of out-of-hospital cardiac arrest outcomes in 27 countries in Europe. Resuscitation. 2016;105:188-95.

6. Deakin CD. The chain of survival: Not all links are equal [Internet]. Resuscitation. 2018. p. 80-2. Available from: http://dx.doi.org/10.1016/j.resuscitation.2018.02.012

7. Brady WJ, Mattu A, Slovis CM. Lay Responder Care for an Adult with Out-of-Hospital Cardiac Arrest [Internet]. New England Journal of Medicine. 2019. p. 2242-51. Available from: http://dx.doi.org/10.1056/nejmra1802529

8. Ro YS, Do Shin S, Song KJ, Hong SO, Kim YT, Cho S-I. Bystander cardiopulmonary resuscitation training experience and self-efficacy of age and gender group: a nationwide community survey [Internet]. The American Journal of Emergency Medicine. 2016. p. 1331-7. Available from: http://dx.doi.org/10.1016/j.ajem.2015.12.001

9. Andrews C, Southworth MK, Silva JNA, Silva JR. Extended Reality in Medical Practice. Curr Treat Options Cardiovasc Med. 2019;21:18.

10. Nas J, Thannhauser J, Vart P, van Geuns R-J, Muijsers HEC, Mol J-Q, et al. Effect of Face-to-Face vs Virtual Reality Training on Cardiopulmonary Resuscitation Quality: A Randomized Clinical Trial. JAMA Cardiol. 2020;5:328-35.

11. Anderson R, Sebaldt A, Lin Y, Cheng A. Optimal training frequency for acquisition and retention of high-quality CPR skills: A randomized trial. Resuscitation. 2019;135:153-61.

12. Bylow H, Karlsson T, Claesson A, Lepp M, Lindqvist J, Herlitz J. Self-learning training versus instructor-led training for basic life support: A cluster randomised trial. Resuscitation. 2019;139:12232. 
13. Roppolo LP, Pepe PE, Campbell L, Ohman K, Kulkarni H, Miller R, et al. Prospective, randomized trial of the effectiveness and retention of 30-min layperson training for cardiopulmonary resuscitation and automated external defibrillators: The American Airlines Study. Resuscitation. 2007;74:276-85.

14. Zapletal B, Greif R, Stumpf D, Nierscher FJ, Frantal S, Haugk M, et al. Comparing three CPR feedback devices and standard BLS in a single rescuer scenario: a randomised simulation study. Resuscitation. 2014;85:560-6.

15. Park S, Lee G. Full-immersion virtual reality: Adverse effects related to static balance. Neurosci Lett. 2020;733:134974.

16. Edelson DP, Sasson C, Chan PS, Atkins DL, Aziz K, Becker LB, et al. Interim Guidance for Basic and Advanced Life Support in Adults, Children, and Neonates With Suspected or Confirmed COVID-19: From the Emergency Cardiovascular Care Committee and Get With The Guidelines-Resuscitation Adult and Pediatric Task Forces of the American Heart Association. Circulation. 2020;141:e933-43.

17. Nolan JP, Monsieurs KG, Bossaert L, Böttiger BW, Greif R, Lott C, et al. European Resuscitation Council COVID-19 guidelines executive summary. Resuscitation. 2020;153:45-55.

18. Craig-Brangan KJ, Day MP. Update: AHA guidelines for CPR and emergency cardiovascular care. Nursing. 2020;50:58-61.

19. Riggs M, Franklin R, Saylany L. Associations between cardiopulmonary resuscitation (CPR) knowledge, self-efficacy, training history and willingness to perform CPR and CPR psychomotor skills: A systematic review. Resuscitation. 2019;138:259-72.

20. Stiell IG, Brown SP, Nichol G, Cheskes S, Vaillancourt C, Callaway CW, et al. What is the optimal chest compression depth during out-of-hospital cardiac arrest resuscitation of adult patients? Circulation. 2014;130:1962-70.

\section{Tables}

Table1. Demographics of participants

\begin{tabular}{|ll|}
\hline & Valid value \\
\hline Age (years) & $18-100$ \\
\hline Sex & Male, Female \\
\hline Weight $(\mathrm{kg})$ & $40-150$ \\
\hline Height $(\mathrm{cm})$ & $100-200$ \\
\hline Previous CPR training & Yes/No \\
\hline
\end{tabular}

Previous CPR training: participants who have previously taken a CPR education

Table2. Test 1 results 
Patients checking and calling for help

Checking for response

Calling for help

Checking for breathing

Chest compression

* The time interval from arrival on the scene to the first chest compression (sec)

Number of chest compressions ( $n$ )

Total $1^{\text {st }} \quad 2^{\text {nd }} \quad 3^{\text {rd }}$ epoch

\begin{tabular}{|c|c|c|c|}
\hline & & & $\begin{array}{l}\text { Valid } \\
\text { value }\end{array}$ \\
\hline & & & $\begin{array}{l}\text { Done/Not } \\
\text { done }\end{array}$ \\
\hline & & & $\begin{array}{l}\text { Done/Not } \\
\text { done }\end{array}$ \\
\hline & & & $\begin{array}{l}\text { Done/Not } \\
\text { done }\end{array}$ \\
\hline $\begin{array}{l}2^{\text {nd }} \\
\text { epoch }\end{array}$ & $\begin{array}{l}3^{\text {rd }} \\
\text { epoch }\end{array}$ & $\begin{array}{l}4^{\text {th }} \\
\text { epoch }\end{array}$ & $\begin{array}{l}\text { Valid } \\
\text { value }\end{array}$ \\
\hline & & & $0-60$ \\
\hline & & & $0-400$ \\
\hline
\end{tabular}

Mean compression depth ( $\mathrm{mm}$ )

$1-100$

Number of adequate compression depth

$0-400$

(n)

Adequate compression depth (\%)

$0-100$

Mean compression rate $(\mathrm{n} / \mathrm{min})$

$1-200$

Adequate compression rate (\%)

$0-100$

Adequate compression depth and rate (\%)

$0-100$

Number of compressions with correct

hand position ( $\mathrm{n}$ )

Correct hand position (\%)

$0-400$

$0-100$

Number of chest compressions with full release $(n)$

Compression and full release (\%)

$0-100$

Hands-off time (sec)

$0-120$

AED

Valid 


\begin{tabular}{|l|c|}
\hline AED use & value \\
\hline Correct AED use & $\begin{array}{l}\text { Done/Not } \\
\text { done }\end{array}$ \\
\hline $\begin{array}{l}\text { *Time from powering on the AED to } \\
\text { defibrillation (sec) }\end{array}$ & Yes/No \\
\hline $\begin{array}{l}\text { *Time from checking for a response to } \\
\text { defibrillation (sec) }\end{array}$ & $0-120$ \\
\hline
\end{tabular}

Epoch, 30-second epochs in Test 1

Adequate compression depth: $5-6 \mathrm{~cm}$, Adequate compression rate: $100-120 / \mathrm{min}$, Correct hand position: hand position as the lower half of the sternum in the centre of the chest, Compression and full release: less than or equal to 5-mm residual displacement, Hands-off time: interruptions of chest compressions during cardiopulmonary resuscitation

* These variables can be obtained from recording devices such as smartphone camera, video camera and so on.

Table3. Test 2 results 
Checking for response

Done/Not done

Calling for help

Done/Not done

Checking for breathing

Done/Not done

Chest compression $\begin{array}{llllll}1^{\text {st }} & 2^{\text {nd }} & 3^{\text {rd }} & 4^{\text {th }} & 5^{\text {th }} & \text { Valid } \\ \text { cycle } & \text { cycle } & \text { cycle } & \text { cycle } & \text { cycle } & \text { value }\end{array}$

*The time interval from arrival on the scene to the first chest compression (sec)

$0-60$

Number of chest compressions ( $n$ )

$0-400$

Mean compression depth ( $\mathrm{mm}$ )

$1-100$

Number of adequate compression depth (n)

$0-400$

Adequate compression depth (\%)

$0-100$

Mean compression rate $(\mathrm{n} / \mathrm{min})$

$1-200$

Adequate compression rate (\%)

$0-100$

Adequate compression depth and rate (\%)

$0-100$

Number of compressions with correct hand position ( $\mathrm{n}$ )

Correct hand position (\%)

Number of chest compressions with full release $(n)$

Compression and full release (\%)

$0-100$

Hands-off time (sec)

$0-120$

AED 
AED use

Done/Not done

Correct AED use

Yes/No

*Time from powering on the AED to $0-120$ defibrillation (sec)

*Time from checking for a response to $0-120$ defibrillation (sec)

Adequate compression depth: 5 - 6 cm, Adequate compression rate: 100 - 120/min, Correct hand position: hand position as the lower half of the sternum in the centre of the chest, Compression and full release: less than or equal to 5-mm residual displacement, Hands-off time: interruptions of chest compressions during cardiopulmonary resuscitation

* These variables can be obtained from recording devices such as smartphone camera, video camera and so on.

\section{Figures}




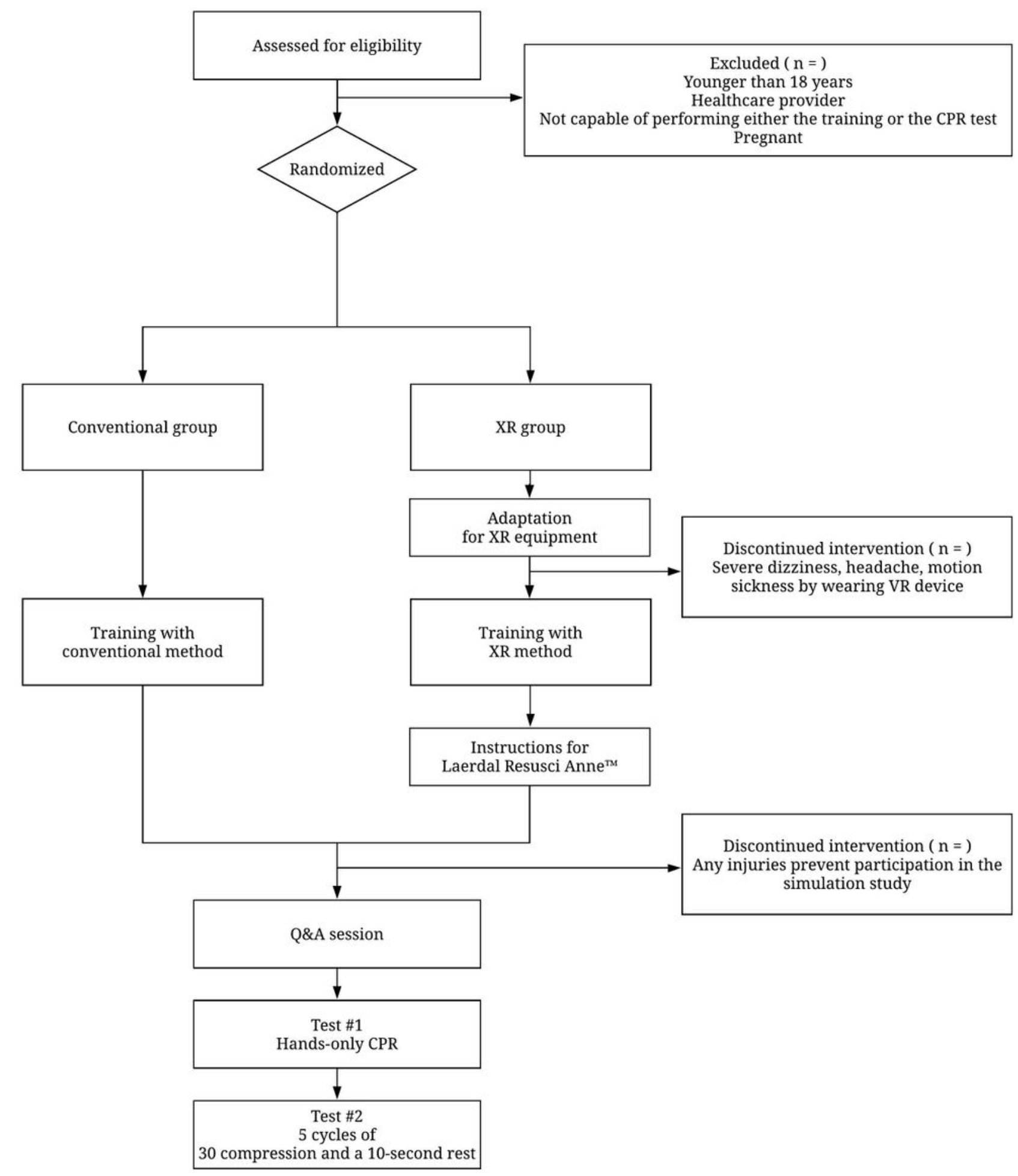

\section{Figure 1}

This study is a prospective, multinational, multicentre, randomized controlled study. Four institutions in 4 countries will participate in the study. The trial protocol will be approved by the Institutional Review Boards of the individual participating institutions (Fig. 1). 


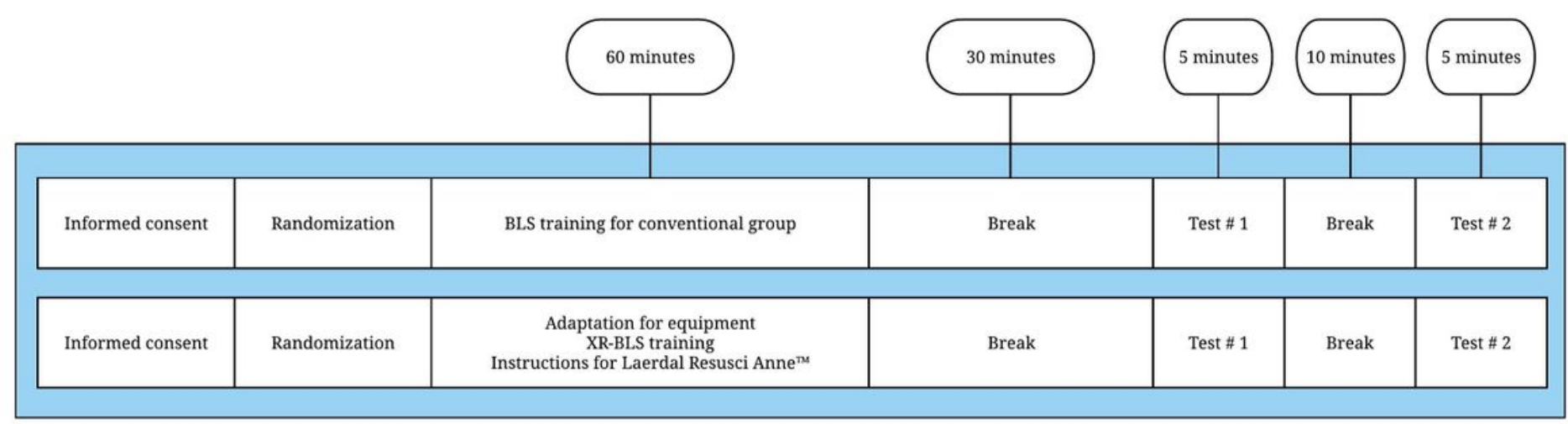

Figure 2

The participant timelines are presented in Fig. 2.

\section{Supplementary Files}

This is a list of supplementary files associated with this preprint. Click to download.

- Supplementaryfile1SPIRIT.docx

- Supplementaryfile2questionnaireConventionalgroup.docx

- Supplementaryfile3questionnaireXRgroup.docx

- Supplementaryfile4InformationSheetConsentForm.docx 